\title{
Variability of Serum Markers of Erythropoiesis during 6 Days of Racing in Highly Trained Cyclists
}

Authors

Affiliations
S. C. Voss ${ }^{1}$, M. Alsayrafi ${ }^{1}$, P. C. Bourdon ${ }^{2}$, F. Klodt ${ }^{3}$, D. Nonis ${ }^{5}$, W. G. Hopkins ${ }^{6}$, Y. O. Schumacher ${ }^{4}$

Affiliation addresses are listed at the end of the article

Key word
doping
EPO
Blood
sera
plasma volume
sTfR

accepted after revision April 17, 2013

Bibliography DOI http://dx.doi.org/ 10.1055/s-0033-1345177 Published online: 2013 Int ] Sports Med (c) Georg Thieme Verlag KG Stuttgart · New York ISSN 0172-4622

Correspondence

\section{Sven Christian Voss}

Blood and EPO Analysis Anti-Doping Lab Qatar P.O.Box 27775

Doha

Qatar

P.O. Box 22287

Tel.: + 974/55/481955

Fax: + 974/4413/2997

s.voss@gmx.de

\section{Abstract}

$\nabla$

The athlete biological passport for the fight against doping is currently based on longitudinal monitoring for abnormal changes in cellular blood parameters. Serum parameters related to altered erythropoiesis could be considered for inclusion in the passport. The aim of this study was to quantify the changes in such parameters in athletes during a period of intense exercise.

12 highly trained cyclists tapered for 3 days before 6 days of simulated intense stage racing. Morning and afternoon blood samples were taken on most days and analysed for total protein, albumin, soluble transferrin receptor and ferritin concentrations. Plasma volume was determined via total haemoglobin mass measured by carbon-monoxide rebreathing. Percent

\section{Introduction}

$\nabla$

The use of erythropoiesis-stimulating agents (ESAs) such as recombinant human erythropoietin (rhEPO), hypoxia-inducible factor (HIF) stabilizers, methoxy polyethylene glycol-epoetin beta (CERA) or other substances with similar chemical structure or biological effects is banned by the World Anti-Doping Agency (WADA) [38].

While there are already direct detection methods available for many substances (i.e., rhEPO), the haematological module of the athlete biological passport (ABP) provides the opportunity to potentially detect the abuse of new substances and methods which are possibly unknown to anti-doping agencies. This indirect, longitudinal approach which is based on monitoring biomarkers of altered erythropoiesis combines haematocrit (Hct), haemoglobin (Hb), red blood-cell count (RBC), reticulocyte percentage (Ret\%), reticulocyte count (Ret\#), mean corpuscular volume (MCV), mean corpuscular haemoglobin changes in means from baseline and percent standard errors of measurement (analytical error plus intra-athlete variation) on each measurement occasion were estimated with mixed linear modelling of log-transformed measures.

Means of all variables changed substantially in the days following the onset of racing, ranging from $-13 \%$ (haemoglobin concentration) to $+27 \%$ (ferritin). After the second day, errors of measurement were generally twice those at baseline.

Plasma variables were affected by heavy exercise, either because of changes in plasma volume (total protein, albumin, haemoglobin), acute phase/inflammatory reactions (ferritin) or both (soluble transferrin receptor). These effects need to be taken into consideration when integrating a plasma parameter into the biological passport model for athletes.

$(\mathrm{MCH})$ and mean corpuscular haemoglobin concentration (MCHC) [32].

Currently only abnormalities in $\mathrm{Hb}$ and OFFScore (calculation based on $\mathrm{Hb}$ and $\mathrm{Ret} \%$ ) are taken into consideration for sanctioning athletes, while the other parameters are used by a panel of independent experts to gain additional information about possible pathological conditions of the athlete or altered sample quality.

The current passport model could be improved with additional markers, however, since inclusion markers require robust and standardized measurement procedures reaching a forensic level of quality. It is obvious that potential new markers must also be highly sensitive to the use of ESAs. Information on the variation of the chosen biomarkers under different conditions and in different populations is also required.

The variables total protein (TP), albumin (Alb), soluble transferrin receptor (sTfR) and ferritin (Fer) are routinely used in medical screenings for which fully automated and highly standardised 
measurement systems are available which help guarantee the validity and robustness of the results. It has been speculated in various studies $[2,4,8]$ that these markers can be used to detect altered erythropoiesis in trials where rhEPO was administered. Notably, in one double-blind, placebo controlled study [4] STfR increased significantly after 1 week of EPO treatment. The same study also showed an increase of the sTfR/Fer ratio, with both changes remaining elevated for 1 week post treatment [4].

In another study on the effects of EPO in athletes and a possible indirect detection in doping control, the authors proposed the use of the sTfR/TP ratio. They argued that iron supplementation makes Fer an unsuitable marker and that the inclusion of TP can be used to adjust for changes in haemoconcentration, which would also be the case for Alb [2]. Furthermore, the intra-individual and day-to-day variation of these parameters have been assessed in several studies [5,7-9,25], however samples were collected from healthy subjects and not athletes who were exposed to intense, high volume exercise.

While information on the influence of gender and age is also available from many medical publications, the information on the variation of these markers in highly trained athletes during periods of heavy exercise is scarce $[1,30]$. An important factor contributing to variability of concentrations of blood constituents is plasma volume (PV). Variations in PV are particularly important in athletes, as changes in their level of physical activity can induce variations in PV of up to $25 \%$, with resulting similar changes in plasma concentrations $[6,13,15,21,22,28]$. The aim of this study was therefore to monitor changes in PV and concentrations of markers in blood that could be used in tests for indirect detection of ESAs in a group of highly trained cyclists taking part in a stage race.

\section{Methods}

$\nabla$

\section{Subjects}

The study was conducted according to the ethical standards outlined in the declaration of Helsinki and approved by the Ethics committee of the University of Freiburg, Germany [16]. The participants were 12 healthy male Caucasian amateur cyclists, who first underwent a full medical check-up with anthropometry to exclude pathological conditions before performing an incremen-

Table 1 Characteristics of the 12 athletes. Data are mean \pm SD.

\begin{tabular}{lr}
\hline age $(\mathrm{y})$ & $27.0 \pm 2.3$ \\
\hline mass $(\mathrm{kg})$ & $69.7 \pm 4.3$ \\
\hline height $(\mathrm{cm})$ & $181.0 \pm 9.7$ \\
\hline body mass index $\left(\mathrm{kg} \cdot \mathrm{m}^{-2}\right)$ & $21.4 \pm 1.5$ \\
\hline body fat $(\%)$ & $12.9 \pm 2.3$ \\
$\mathrm{VO}_{2}$ max $\left(\mathrm{ml} \cdot \mathrm{kg}^{-1} \cdot \mathrm{min}^{-1}\right)$ & $63.8 \pm 5.7$ \\
haemoglobin $\left(\mathrm{g} \cdot \mathrm{dL}^{-1}\right)$ & $14.8 \pm 0.7$ \\
haematocrit $(\%)$ & $42.9 \pm 2.3$ \\
haemoglobin mass $(\mathrm{g})$ & $864 \pm 81$
\end{tabular}

tal cycling test until exhaustion for the determination of their maximal oxygen uptake $\left(\mathrm{VO}_{2} \mathrm{max}\right)$. Their anthropometric, fitness and simple haematological data are presented in $\bullet$ Table 1.

\section{Cycling intervention}

The investigation took place in Qatar. To mimic a real training and competition regime, the subjects underwent 3 days of reduced training after the air travel from Germany to Qatar, followed by a 6-day cycling stage race simulation. The simulated cycling stage race was designed to be as close to a real race as possible and required certain tasks to be performed by the participants every day additional to or partly replacing the 3-4h of daily road cycling. Special primes were available as incentive to fulfil the daily goals. Table $\mathbf{2}$ presents the information on the stages of the 6 exercise days including distance, specific tasks and ambient temperature during exercise.

\section{Blood sampling}

Venous blood sampling was performed according to the current WADA guidelines for the ABP (blood sampling) [37]. Blood was drawn in a sitting position with the subjects having remained seated for at least $10 \mathrm{~min}$ to avoid fluctuations in PV. Sampling took place every morning at 8:00 a.m. before breakfast and every evening at 6:00 p.m., which was at least $2 \mathrm{~h}$ post exercise. A volume of $11.7 \mathrm{ml}$ of blood was obtained on each occasion (one $2.7 \mathrm{ml}$ EDTA tube (Sarstedt, Numbrecht, Germany) and one $9.0 \mathrm{ml}$ tube with clot activator (S-Monovette, Sarstedt, Numbrecht, Germany)).

\section{Haemoglobin mass and plasma volume}

To investigate PV effects, $\mathrm{Hb}$ mass was measured using the optimised CO rebreathing method as previously described by Schmidt and Prommer [29]. Hb mass was measured in duplicate in each subject at the beginning of the study. The true $\mathrm{Hb}$ mass was calculated for each time point taking into account the exact loss of $\mathrm{Hb}$ due to blood sampling. Plasma volume for each sampling time point was then calculated from $\mathrm{Hb}$ mass, Hb concentration and Hct (see calculations and statistics). Reticulocyte percentage, tested daily to prevent a misinterpretation of our data caused by an increased Hb synthesis, did not show any changes. The analytical error of measurement of the $\mathrm{Hb}$ mass measurements calculated from the duplicate measures was $2.3 \%$ [18].

\section{Haematological parameters}

The EDTA blood samples were analysed within 90 min of collection on a Sysmex XT-2000i haematology analyser (Sysmex Corp., Kobe, Japan) according to WADA guidelines. The analytical error of measurement as a coefficient of variation for $\mathrm{Hb}$ and Hct were $0.3 \%$ and $0.4 \%$ respectively.

Table 2 Information about the simulated race stages.

\begin{tabular}{|c|c|c|c|c|c|c|}
\hline & Stage 1 & Stage 2 & Stage 3 & Stage 4 & Stage 5 & Stage 6 \\
\hline study day & 4 & 5 & 6 & 7 & 8 & 9 \\
\hline distance $(\mathrm{km})$ & 124 & 136 & 152 & 152 & 45 & 124 \\
\hline duration (h) & $3: 22$ & 4:00 & $4: 14$ & $4: 12$ & $0: 49$ & 4:05 \\
\hline special tasks & 3 sprints & time-trial & - & 3 sprints & time trial & criterium \\
\hline ambient temperature $\left({ }^{\circ} \mathrm{C}\right)$ & 27 & 24 & 23 & 24 & 25 & 27 \\
\hline
\end{tabular}




\section{Serum parameters}

The sera samples were centrifuged within $30 \mathrm{~min}$ after collection, aliquoted and stored at $-20^{\circ} \mathrm{C}$ for batch analysis. All parameters were then analysed 4 weeks after the study on a Roche Cobas Integra 400 plus analyser (Roche, Mannheim, Germany). The analytical error of measurement, estimated in an in-house validation study, was $0.9 \%$ for TP, $1.3 \%$ for Alb, $2.0 \%$ for sTfR and $5.1 \%$ for Fer.

All samples were analysed for TP and Alb. However, due to reagent costs sTfR and Fer were analysed for all morning samples but not for all evening samples. Evening sTfR analysis was performed on Days 1, 2, 6, 8, while for Fer evening samples were tested on Days 1, 2, 6, 7, 8 .

\section{Calculations}

$\mathrm{Hb}$ mass was calculated on the assumption that the mean of the $2 \mathrm{Hb}$ mass tests on Days 2 and 3 of the study represented a stable initial value. Every time blood was sampled the corresponding $\mathrm{Hb}$ mass was calculated using the following formula:

Actual $\mathrm{Hb}$ mass $(\mathrm{g})=$ last $\mathrm{Hb}$ mass $(\mathrm{g})-(0.117 \times$ measured $\mathrm{Hb}$ $\left(\right.$ g. $\left.\left.\mathrm{dl}^{-1}\right)\right)$.

The factor 0.117 was used to correct for the $11.7 \mathrm{ml}$ drawn during each blood sample. Assuming a random error of as much as $\pm 1 \mathrm{ml}$ at each withdrawal, the accumulated error after 12 withdrawals by the last withdrawal of the stage race would be $\pm 1 \times \sqrt{12}= \pm 3.5 \mathrm{ml}$; for a $\mathrm{Hb}$ concentration of $15 \mathrm{~g} \cdot \mathrm{dl}^{-1}$ and final $\mathrm{Hb}$ mass of $840 \mathrm{~g}$, the resulting error in $\mathrm{Hb}$ mass would be $\pm 0.5 \mathrm{~g}$ or $0.06 \%$, which is negligible compared with the observed errors of measurement for blood parameters.

\section{Statistics}

The aim of the analysis was to estimate the systematic bias and random error for measurements of specific blood parameters during periods of intense exercise, such as cycling stage racing. The errors of measurement were estimated to include analytical error and real intra-subject (or biological) changes, because these are the errors that a practitioner or researcher has to contend with when tracking changes in an individual or group. The last 2 morning measurements before the first race (i.e., the mornings of Days 3 and 4) were used as a stable baseline for the estimation of error of measurement (intra-subject variability) at other measurement times. Changes in the mean at other times were calculated as changes from the morning of Day 4. (For sTfR, Days 2 and 3 were chosen as baseline, and changes in means were calculated from Day 3, owing to greater error on the morning of Day 4). A separate analysis was performed for each of the other days of measurements by including it in a mixed linear model with the 2 baseline days.

The analyses were realized with Proc Mixed in the Statistical Analysis System (Version 9.2, SAS Institute, Cary NC). The only fixed effect in the model had 6 levels to estimate the mean of the parameter on each morning (pre-racing) and afternoon (postracing) of the 3 days. The random effects were cyclist identity (to estimate each cyclist's mean), interactions of cyclist identity with dummy variables having values of 1 for each afternoon of the 2 baseline days and for the morning and afternoon of the other day and 0 otherwise (to estimate extra measurement error at those measurement times) and the residual (to estimate error of measurement in the baseline morning measurements). The error of measurement (intra-subject variability) at times other than morning baseline was estimated by adding the residual variance to the corresponding extra variance and taking the square root. Negative values for the extra variance were permitted for the purpose of properly allowing for the possibility that sampling variation could result in errors of measurement smaller on the afternoons of baseline days and on the other days than those on baseline mornings. The error of measurement at baseline was estimated by taking the square root of the mean of the residual variance from all analyses. An estimate of the standard deviation of change scores between 2 baseline tests and between a baseline test and the test with maximum error was derived by adding the error variances of the 2 tests and taking the square root.

Changes in the mean and errors of measurement were expressed in per cent units by back-transformation following analysis of the log-transformed values of the parameter [19]. The appropriateness of log transformation for each dependent variable was checked by inspection of plots of residual vs. predicted values, which showed no evidence of non-uniformity. Confidence limits for changes in the mean were provided by the mixed modelling procedure. Confidence limits for the errors of measurement were approximations based on the assumption that the sampling distribution of their variances was normal. Outcomes were assessed inferentially on the basis of the uncertainty represented by the confidence interval [19].

\section{Results}

$\nabla$

The mean changes and errors of measurement (intra-subject variability) in blood parameters over the duration of the study are shown in $\bullet$ Fig. 1. Table 3 shows the baseline mean and standard deviations of the parameters as well as measures relevant to testing for changes in individual athletes: the maximum changes in the means between a baseline test and test during the stage race, the standard deviations of change scores between tests at baseline, and the maximum standard deviations of change scores between a baseline test and a test during the stage race. Given the uncertainty in the estimates (95\% confidence limits), all of the maximum mean changes were clearly substantial, and most of the maximum standard deviations of change scores were clearly greater than those at baseline.

\section{Discussion \\ $\nabla$}

All the measured sera variables were affected substantially by the stage race. The magnitude of the change varied between the parameters, as illustrated in $\bullet$ Fig. 1. The PV showed a small decrease during the tapering period, followed by an increase during the race days. Timelines for morning and evening were very similar with the exception of a small drop on Day 9 for the evening values. Total protein, Alb and sTfR showed a continuous decrease for the morning values. Ferritin started to increase from Day 5 and decreased again after Day 7. All parameters also showed a substantial increase in the standard error of measurement (intra-subject variability), starting from the third race day (Day 6). The maximum error occurred between the third and sixth race day, depending on the parameter.

In $\bullet$ Fig. 1 there is an apparent relationship between high PV and high standard error. Interestingly the standard error for Alb on Day 1 is also relatively high. A possible reason might be the 
residual effect of the air travel, which could have stimulated albumin synthesis as described by Imoberdorf et al. [20]. In their study an increase in absolute synthesis rate and Alb concentration was observed in a group of subjects who were transported by helicopter to an altitude of $4500 \mathrm{~m}$. Imoberdorf et al. [20]

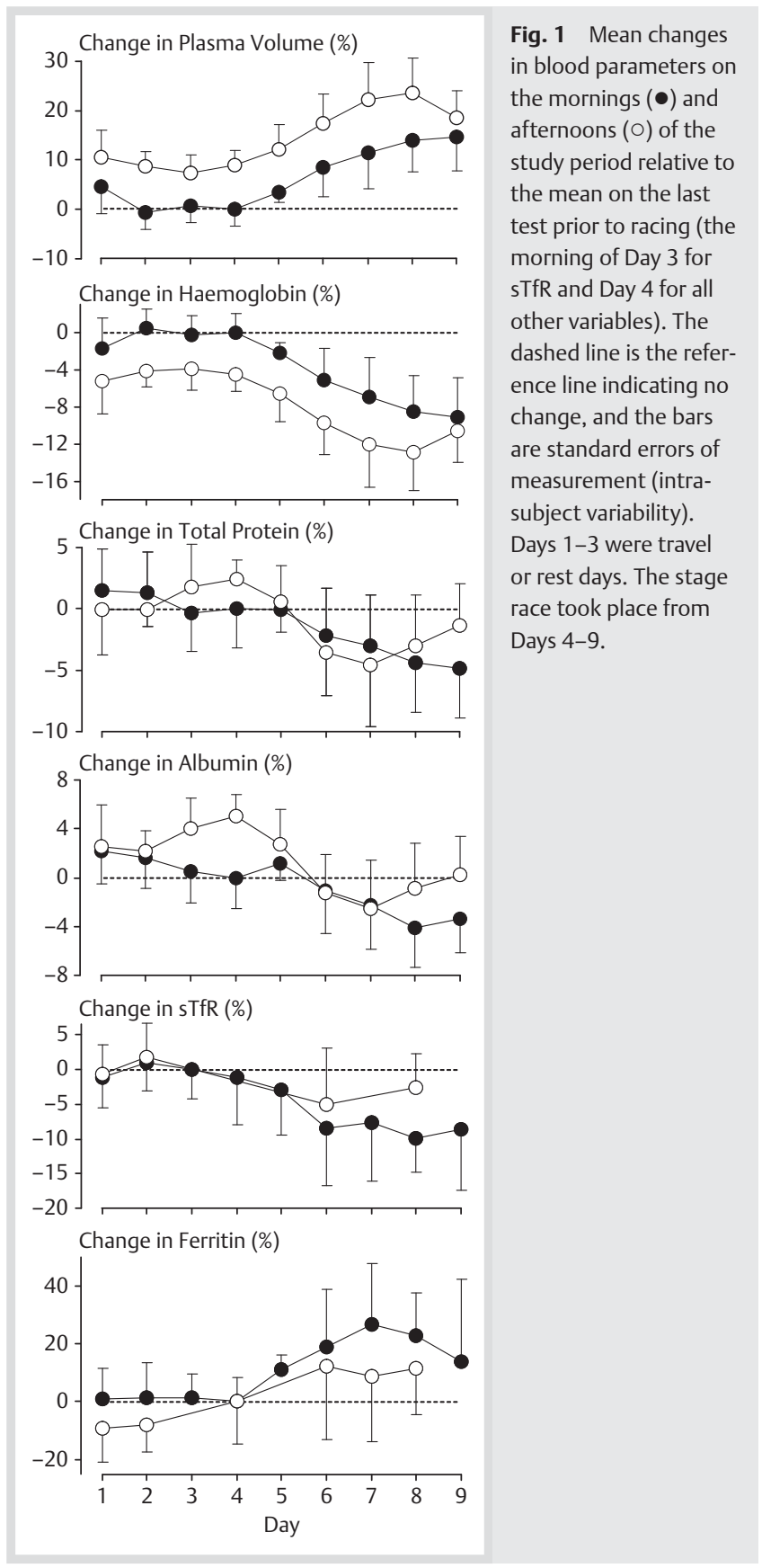

speculated that the increase in Alb synthesis could result from respiratory alkalosis, which would compensate for the negative effects of hypoxia on protein biosynthesis. Another reason could be the influence of the PV. $\diamond$ Fig. 1 shows that the standard error for PV was also higher for Day 1 when compared to Days 2 and 3. The PV can be affected by hydration, and as Alb regulates the BV by maintaining the oncotic pressure, this could provide another explanation for the higher error.

The increase in the error for the PV can be explained by individual adaptations to the stage racing and to the environmental conditions with on average $17^{\circ} \mathrm{C}$ higher temperatures than in the country of origin. Both exercise and thermoregulation are known to increase PV [14]. Due to the characteristics of a cycling competition, the workload of the subjects was not standardized as in a laboratory investigation, and thus differences related to the individual daily form, effort, strategy and motivation may have also influenced this error. However, $\bullet$ Fig. 1 clearly demonstrates the increase of the PV during the Race Days (Days 4-9). The diurnal variation in PV, which can be observed in $\bullet$ Fig. 1, is also in agreement with previous studies $[26,36]$. This variation is mirrored by an increase of $\mathrm{Hb}$ concentration and Hct (not shown) due to dehydration overnight. The increase in total PV seems however not to affect this diurnal rhythm [13]. As Hb is a parameter that is already used in anti-doping, our data on the dependence of the $\mathrm{Hb}$ concentration on plasma volume changes ( $\bullet$ Fig. 1, Table 3) also highlight one of the biggest challenges in this endeavour: interpretation of an individual biological profile must take into account the recent training and competition experience of the athlete. Currently WADA is addressing this issue in their sampling reports, and there are a limited number of specialists who perform these interpretations.

The mean changes of TP and Alb were below $5 \%$. The reduction during the Race Days demonstrated in $\bullet$ Fig. 1 could be mainly due the PV expansion. However, as Alb is also a negative acute phase protein another possible reason could be exercise-induced inflammatory processes [20]. This hypothesis finds support in $\bullet$ Fig. 1, which shows a mean increase of Fer during the race of $\sim 20 \%$. Ferritin is also a positive acute phase protein that is upregulated with inflammation [35]. As all subjects were healthy and not iron deficient, an increase in Fer caused by changes in nutrition can be excluded.

Total protein has been proposed in several studies as a marker to correct for PV changes [2,33]. While this approach might provide valid results in highly standardized non-exercise settings for measurements within a few hours, our results show that it is not possible to use this parameter in an anti-doping context over a period of several days. Both TP and Alb showed a time course that did not closely follow the time course of PV. During the later stages of the study both of these parameters showed an inconsistency in their values within the same day as well as an

Table 3 Means and SD of blood parameters at baseline, and changes in values relevant to blood testing (with $95 \%$ confidence limits) derived from the data in Fig. 1: maximum changes in the means during the stage race, standard deviations of change scores between baseline tests, and maximum standard deviations of change scores between a baseline test and a test during the stage race.

\begin{tabular}{|c|c|c|c|c|}
\hline & Baseline (mean \pm SD) & Maximum change in mean (\%) & Baseline SD of change scores (\%) & Maximum SD of change scores (\%) \\
\hline plasma volume & $3.34 \pm 0.45 \mathrm{~L}$ & $+24 ; \pm 5$ & $4.9 ; \pm 2.3$ & $8.4 ; \pm 4.0$ \\
\hline haemoglobin & $148.0 \pm 6.6 \mathrm{~g} \cdot \mathrm{L}^{-1}$ & $-13 ; \pm 2.2$ & $2.8 ; \pm 1.3$ & $5.1 ; \pm 2.5$ \\
\hline total protein & $69.7 \pm 4.6 \mathrm{~g} \cdot \mathrm{L}^{-1}$ & $-4.8 ; \pm 2.7$ & $4.5 ; \pm 1.4$ & $5.9 ; \pm 2.6$ \\
\hline albumin & $47.0 \pm 2.2 \mathrm{~g} \cdot \mathrm{L}^{-1}$ & $-4.1 ; \pm 2.0$ & $3.7 ; \pm 1.3$ & $4.7 ; \pm 2.3$ \\
\hline ferritin & $77 \pm 51 \mathrm{ng} \cdot \mathrm{ml}^{-1}$ & $+27 ; \pm 14$ & $11.6 ; \pm 5.9$ & $30 ; \pm 16$ \\
\hline sTfR & $2.75 \pm 0.62 \mathrm{mg} \cdot \mathrm{L}^{-1}$ & $-10 ; \pm 3.4$ & $5.8 ; \pm 3.0$ & $9.7 ; \pm 4.7$ \\
\hline
\end{tabular}

STfR, soluble transferrin receptor 
increase in error with the change in PV due to exercise. Total protein for example shows higher mean values in the morning than in the evening on some days (i.e., Days 6 and 7) and the opposite on others days (i.e., Days 8 and 9). Immunological processes during an infection, which down-regulate Alb or increase the TP concentration by the production of immunoglobulin must also be kept in mind.

Another marker which we investigated was Fer. Ferritin is highly affected by the abuse of rhEPO and by blood transfusion. In such settings, Fer will typically decrease during abuse of ESAs or following blood withdrawal and increase after blood transfusion. Although it can easily be influenced by iron supplementation, it is nevertheless a marker with a high sensitivity for blood manipulation. Our study highlights however the role of Fer as an acute phase protein, as discussed above $[11,12,34]$. Ferritin increased with on-going race duration up to Day 7 . While a decrease was then observed starting from Day 8, the mean values remained higher when compared to the Resting Days. The decrease could have resulted from a combination of declining inflammatory processes and/or an increased iron demand. Harris et al. [17] stated that one of the main requirements of a biological marker for longitudinal follow ups is that the intra-individual variability should be lower than the corresponding inter-individual variability. In the current literature for biological variation, the intersubject biologic variation of Fer is listed with $15.0 \%$ while the intra-subject variation has a value of $14.2 \%$ [27]. In our study we saw an increase in error from $8.2 \%$ during the resting period to nearly $30 \%$ as a result of high-intensity and high-volume exercise. Thus, from a purely mathematical point of view, Fer has certain limitations as a future marker for the haematological module of the ABP during periods of high intensity exercise. On the other hand its high sensitivity for blood manipulation has been reported in several studies, and the magnitude of changes in Fer observed after typical blood manipulations such as blood withdrawal and reinfusion are usually much higher than those induced by exercise [3]. Furthermore, the direction of changes (increase/decrease) is an element of interpretation depending on the timing of the blood test in relation to the training and competition schedule of the athlete. Therefore, Fer might add valuable information in the hands of skilled experts who are able to discern inflammatory and exercise related effects from the results of blood manipulation.

The most stable marker in our study was the sTfR, the circulating, proteolytic product of the transferrin receptor that is expressed on cells depending on the iron demand and rate of erythropoiesis. The sTfR concentration in plasma is proportional to the cellular transferrin receptor. As demonstrated in $\bullet$ Fig. 1, the time course of the sTfR morning values during our study seems to be inversely related to the time course of the PV (i.e., when the PV increases, the sTfR concentration decreases). As described in several studies, sTfR is not influenced by inflammation $[1,23,24,30]$, and thus a potential increase in concentrations during periods of heavy exercise would either reflect a possible dehydration or an increase in erythropoiesis. One shortcoming of our study in this context was the lack of regular daily evening measurements. We do not have an explanation for the slightly higher values in the evening of Day 6 and 8 . We can only presume that the difference in the error might be related to the exercise load on these days or due to analytical batch variability of the reagents. Abellan et al. [1] reported the inter-assay variability for the assay used in our study as below $2 \%$, which is relatively low. We did not find information in the literature that sTfR exhibits a significant diurnal variation. While Schumacher et al. also demonstrated that immediate exercise has no significant effect on the transferrin receptor [30], Abellan et al. reported a significant increase of sTfR after a 21-km running race but not after a $10-\mathrm{km}$ race. Therefore, the accumulation of exercise load during our study or the time of blood sampling ( $2 \mathrm{~h}$ after exercise) perhaps had an influence on the sTfR values. In our study we found a change in the error from $4.4 \%$ at baseline to $8.1 \%$ during the stage race simulation, which is still much lower compared to an inter-individual variation of $35 \%$ which we observed in a reference population of 200 adolescent athletes (unpublished data). The $8.1 \%$ variability we reported is also in line with the data of Abellan who found an intra-athlete variability of $9 \%$ when analysing blood samples of athletes from different sports over the period of a complete training and competition season [1]. Due to its relatively low variability, sTfR seems to be a possible candidate for the inclusion in the haematological module of the biological passport. Another advantage of this marker in serum is sufficient stability for changes that occur during transport of samples to be negligible [10]. However before implementation, it would have to be decided which analytical technique should be applied for the analysis of the sTfR, as the lack of standardization in the analysis of this parameter is still an issue and might cause differences in analytical results between laboratories.

\section{Conclusion \\ $\nabla$}

Nearly all of the plasma variables assessed were significantly affected by heavy exercise. This can be either related to PV-associated processes (TP, Alb) or to acute phase/inflammatory reactions (Fer) or both. However the same dependence on PV is also relevant for $\mathrm{Hb}$, which is already one of the key parameters in the blood passport. Thus before integrating a new plasma parameter into the Athletes Biological Passport model, the described effects need to be taken into consideration.

\section{Acknowledgements \\ $\nabla$}

The authors want to thank Noora Alsowaidi and Mohamed Ibrahim Elzain Elgingo for helping in the project preparation and daily sample collections.

\author{
Affiliations \\ Anti-Doping Lab Qatar, Doha, Qatar \\ Aspire Academy, Sport Science, Doha, Qatar \\ ${ }^{3}$ University of Freiburg, Germany \\ ${ }^{4}$ Aspetar - Qatar Orthopaedic and Sports Medicine Hospital Doha, Qatar \\ Exercise and Sport Sciences, Università di Milano, Italy \\ ${ }^{6}$ Sport Performance Research Institute of New Zealand, AUT University, \\ Auckland, New Zealand
}

\section{References}

1 Abellan R, Ventura R, Pichini S, Palmi I, Bellver M, Olive R, Pacifici R, Pascual JA, Zuccaro P, Segura J. Effect of physical fitness and endurance exercise on indirect biomarkers of recombinant erythropoietin misuse. Int J Sports Med 2007; 28: 9-15

2 Audran M, Gareau R, Matecki S, Durand F, Chenard C, Sicart MT, Marion $B$, Bressolle $F$. Effects of erythropoietin administration in training athletes and possible indirect detection in doping control. Med Sci Sports Exerc 1999; 31: 639-645 
3 Berglund B, Birgegård G, Wide L, Pihlstedt P. Effects of blood transfusions on some hematological variables in endurance athletes. Med Sci Sports Exerc 1989; 21: 636-642

4 Birkeland KI, Stray-Gundersen J, Hemmersbach P, Hallen J, Haug E, Bahr $R$. Effect of rhEPO administration on serum levels of sTfR and cycling performance. Med Sci Sports Exerc 2000; 32: 1238-1243

5 Borel MJ, Smith SM, Derr J, Beard JL. Day-to-day variation in iron-status indices in healthy men and women. Am J Clin Nutr 1991; 54: 729-735

6 Convertino VA. Blood volume response to physical activity and inactivity. Am J Med Sci 2007; 334: 72-79

7 Cooper MJ, Zlotkin SH. Day-to-day variation of transferrin receptor and ferritin in healthy men and women. Am J Clin Nutr 1996; 64: 738-742

8 Costongs GM, Janson PC, Bas BM, Hermans J, Brombacher PJ, van Wersch $J W$. Short-term and long-term intra-individual variations and critical differences of haematological laboratory parameters. J Clin Chem Clin Biochem 1985; 23: 69-76

9 Dale JC, Burritt MF, Zinsmeister AR. Diurnal variation of serum iron, iron-binding capacity, transferrin saturation, and ferritin levels. Am J Clin Pathol 2002; 117: 802-808

10 Drammeh BS, Schleicher RL, Pfeiffer CM, Jain RB, Zhang M, Nguyen PH. Effects of delayed sample processing and freezing on serum concentrations of selected nutritional indicators. Clin Chem 2008; 54 : 1883-1891

11 Fallon KE. The acute phase response and exercise: the ultramarathon as prototype exercise. Clin J Sport Med 2001; 11: 38-43

12 Fallon KE, Sivyer G, Sivyer K, Dare A. Changes in haematological parameters and iron metabolism associated with a 1600 kilometre ultramarathon. Br J Sports Med. 1999; 33: 27-31

13 Fellmann $N$. Hormonal and plasma volume alterations following endurance exercise. A brief review. Sports Med 1992; 13: 37-49

14 Garrett AT, Rehrer NJ, Patterson MJ. Induction and decay of short-term heat acclimation in moderately and highly trained athletes. Sports Med 2011; 41: 757-771

15 Greenleaf JE, Convertino VA, Mangseth GR. Plasma volume during stress in man: osmolality and red cell volume. J Appl Physiol 1979; 47: $1031-1038$

16 Harriss DJ, Atkinson G. Update - ethical Standards in Sport and Exercise Science Research. Int J Sports Med 2011; 32: 819-821

17 Harris EK. Effects of intra- and interindividual variation on the appropriate use of normal ranges. Clin Chem 1974; 20: 1535-1542

18 Hopkins WG. Measures of reliability in sports medicine and science. Sports Med 2000; 30: 1-15

19 Hopkins WG, Marshall SW, Batterham AM, Hanin J. Progressive statistics for studies in sports medicine and exercise science. Med Sci Sports Exerc 2009; 41: 3-13

20 Imoberdorf R, Garlick PJ, McNurlan MA, Casella GA, Peheim E, Turgay M, Bärtsch $P$, Ballmer PE. Enhanced synthesis of albumin and fibrinogen at high altitude. J Appl Physiol 2001; 90: 528-537

21 Kargotich S, Goodman C, Keast D, Fry RW, Garcia-Webb P, Crawford PM, Morton AR. Influence of exercise-induced plasma volume changes on the interpretation of biochemical data following high-intensity exercise. Clin J Sport Med 1997; 7: 185-191

22 Kargotich S, Goodman C, Keast D, Morton AR. The influence of exerciseinduced plasma volume changes on the interpretation of biochemical parameters used for monitoring exercise, training and sport. Sports Med 1998; 26: 101-117

23 Marković M, Majkić-Singh N, Ignjatović S, Singh S. Reticulocyte haemoglobin content vs. soluble transferrin receptor and ferritin index in iron deficiency anaemia accompanied with inflammation. Int J Lab Hematol 2007; 29: 341-346
24 Nikolaidis MG, Michailidis Y, Mougios V. Variation of soluble transferrin receptor and ferritin concentrations in human serum during recovery from exercise. Eur J Appl Physiol 2003; 89: 500-502

25 Pilon VA, Howanitz PJ, Howanitz JH, Domres N. Day-to-day variation in serum ferritin concentration in healthy subjects. Clin Chem 1981; 27: $78-82$

26 Pocock SJ, Ashby D, Shaper AG, Walker M, Broughton PM. Diurnal variations in serum biochemical and haematological measurements. J Clin Pathol 1989; 42: 172-179

27 Ricós C, Alvarez V, Cava F, García-Lario JV, Hernández A, Jiménez CV Minchinela J, Perich C, Simón M. Current databases on biological variation: pros, cons and progress. Scand J Clin Lab Invest 1999; 59: 491-500

28 Sawka MN, Convertino VA, Eichner ER, Schnieder SM, Young AJ. Blood volume: importance and adaptations to exercise training, environmental stresses, and trauma/sickness. Med Sci Sports Exerc 2000; 32: 332-348

29 Schmidt $W$, Prommer $N$. The optimised CO-rebreathing method: a new tool to determine total haemoglobin mass routinely. Eur J Appl Physiol 2005; 95: 486-495

30 Schumacher YO, Schmid A, König D, Berg A. Effects of exercise on soluble transferrin receptor and other variables of the iron status. $\mathrm{Br}$ J Sports Med 2002; 36: 195-199

31 Schumacher YO, Temme J, Bueltermann D, Schmid A, Berg A. The influence of exercise on serum markers of altered erythropoiesis and the indirect detection models of recombinant human erythropoietin abuse in athletes. Haematologica 2003; 88: 712-714

32 Sottas PE, Robinson N, Saugy M. The athlete's biological passport and indirect markers of blood doping. Handb Exp Pharmacol 2010; 305-326

33 Strauss MB, Davis RK, Rosenbaum JD, Rossmeisl EC. Water diuresis produced during recumbency by the intravenous infusion of isotonic saline solution. J Clin Invest 1951; 30: 862-868

34 Taylor C, Rogers G, Goodman C, Baynes RD, Bothwell TH, Bezwoda WR Kramer F, Hattingh J. Hematologic, iron-related, and acute-phase protein responses to sustained strenuous exercise. J Appl Physiol 1987; 62: 464-469

35 Thurnham DI, McCabe LD, Haldar S, Wieringa FT, Northrop-Clewes CA, McCabe GP. Adjusting plasma ferritin concentrations to remove the effects of subclinical inflammation in the assessment of iron deficiency: a meta-analysis. Am J Clin Nutr 2010; 92: 546-555

36 Touitou Y, Touitou C, Bogdan A, Reinberg A, Auzeby A, Beck H, Guillet $P$. Differences between young and elderly subjects in seasonal and circadian variations of total plasma proteins and blood volume as reflected by hemoglobin, hematocrit, and erythrocyte counts. Clin Chem 1986; 32: 801-804

37 WADA. Athlete Biological Passport Operating Guidelines 2012; Available from: http://www.wada-ama.org/Documents/Science_Medicine/ Athlete_Biological_Passport/WADA-ABP-Operating-Guidelines_v3.1EN.pdf

38 WADA. The 2013 Prohibited List International Standard. Available from: http://www.wada-ama.org/Documents/World_Anti-Doping_Program/ WADP-Prohibited-list/2013/WADA-Prohibited-List-2013-EN.pdf 